\title{
High follicle-stimulating hormone levels should not necessarily lead to the exclusion of subfertile patients from treatment
}

\author{
Ilse A. J. van Rooij, Ph.D., ${ }^{a}$ Evelyn de Jong, M.D., ${ }^{a}$ Frank J. M. Broekmans, Ph.D., ${ }^{a}$

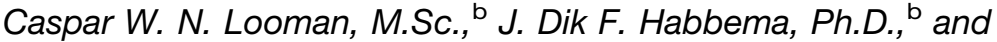 \\ Egbert R. te Velde, Ph.D. ${ }^{a}$
}

University Medical Center Utrecht, Utrecht, The Netherlands

Objective: To determine ongoing pregnancy rates in subfertile patients with elevated FSH levels and regular cycles and to assess whether or not it is justified to exclude such patients from treatment on the basis of elevated FSH levels alone.

Design: Retrospective follow-up study.

Setting: Tertiary fertility center.

Patient(s): One hundred twenty-two patients with normal FSH levels $<10.0$ IU/L, 126 with FSH between 10.0 and 15.0 IU/L, and 53 with FSH levels $>15.0 \mathrm{IU} / \mathrm{L}$, all having regular cycles and belonging to a general subfertility population.

Intervention(s): Follow-up.

Main Outcome Measure(s): Overall and treatment-independent and treatment-dependent ongoing pregnancy rates and time to ongoing pregnancy.

Result(s): Overall ongoing pregnancy rates declined from $65 \%$ in the normal FSH group to $47 \%$, and $28 \%$ in the respective elevated FSH groups. However, when adjusting for differences in age and whether or not treatment was applied, this declining trend became inconsistent for both treatment-independent and treatmentdependent ongoing pregnancy rates. Only when FSH levels exceeded $20 \mathrm{IU} / \mathrm{L}$ was a clear fall in ongoing pregnancy rate observed, independent of age. In a Cox regression analysis, FSH seemed significantly associated with the outcome time to overall ongoing pregnancy (odds ratio $=0.94,95 \%$ confidence interval, $0.88-0.99$ ), but after adjusting for age and being on treatment or not this significance disappeared (odds ratio $=0.97,95 \%$ confidence interval, 0.91-1.01).

Conclusion(s): The contribution of FSH in the initial evaluation of subfertile couples is restricted to counseling patients on the probability of having lower chances of conceiving. It does not seem justified to exclude patients with normal regular cycles from treatment on the basis of the FSH value alone. (Fertil Steril ${ }^{\circledR}$ 2004;81:1478-85. (C2004 by American Society for Reproductive Medicine.)

Key Words: FSH, subfertility, pregnancy, regular cycle

With increasing female age, the probability of pregnancy declines $(1,2)$. This decline in fertility is most probably due to diminishing ovarian reserve, that is, the quantity and quality of the follicles in the ovary (3). However, biological variation exists in the ovarian reserve of women of the same age, and therefore chronological age alone may not give a reliable reflection of ovarian reserve.

These observations led to the search for ovarian reserve tests, of which the measure- ment of FSH in the early follicular phase is the most common one. The value of FSH has been mainly tested in IVF populations. Those IVF patients with elevated FSH levels were shown to have lower pregnancy rates $(4,5)$, and the predictive value of FSH was better than age alone (6).

Recently, some debate about the real clinical value of measuring FSH before IVF has arisen because investigators have found that FSH is of limited value in predicting ongoing 
Inclusion of patients and patient management and outcome stratified by FSH classes.

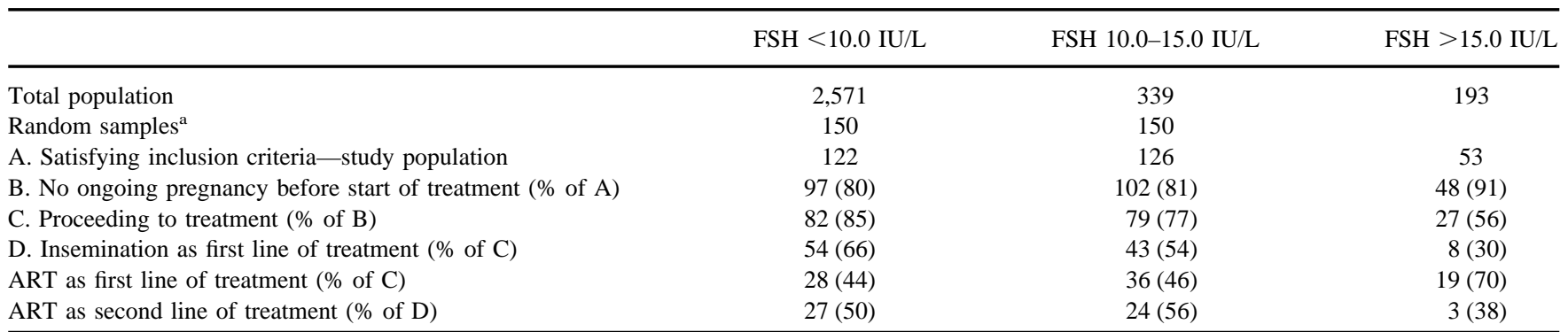

Note: $\mathrm{ART}=$ assisted reproductive technologies.

${ }^{a}$ Random samples were taken from patients within FSH classes $<10.0$ IU/L and 10.0-15.0 IU/L.

Van Rooij. Elevated FSH level and pregnancy. Fertil Steril 2004.

pregnancy in IVF patients (7), that age was more predictive for the occurrence of pregnancy after IVF (8), and that moderately elevated FSH levels were not predictive of IVF outcome, especially in younger women (9). In contrast to these findings, El-Toukhy et al. (10) concluded that younger women with elevated FSH levels above $10 \mathrm{IU} / \mathrm{L}$ had lower implantation rates after IVF.

In a general subfertility population the prevalence of the "disease" (nonpregnancy) is lower. This leads to a decline in the positive predictive value, that is, the probability of indeed not becoming pregnant when patients have an abnormal FSH level (11). If the consequence of having an abnormal test is exclusion from treatment, an increased number of patients with a false-positive test will occur and consequently will not be treated optimally.

In the past years a few reports on the value of FSH in general infertility patients have been published with contrasting results. Levi et al. (12) showed low conception (5\%) and high fetal loss rates in a group of infertile patients with elevated FSH levels. According to this report, patients with diagnosed diminished ovarian reserve should be advised not to start infertility treatment. However, the investigators coupled FSH and hCG results of a laboratory database and did not provide information on patient characteristics and type of management and did not correct for the duration of followup. Other studies in general subfertility populations show comparable pregnancy rates between patients with normal and elevated FSH levels (13), especially when patients are younger (14). Therefore, it has become questionable whether patients with elevated FSH levels should be excluded from treatment regardless of the other diagnostic or prognostic features.

The measurement of basal FSH levels is a standard procedure in the diagnostic workup of patients in our clinic. In this retrospective study we evaluated overall, treatmentindependent, and treatment-dependent ongoing pregnancy rates of patients with a regular cycle and with raised $\mathrm{FSH}$ levels in the initial diagnostic workup. The main aim was to find out whether patients from a general subfertility population with elevated FSH levels should be excluded from treatment. Furthermore, we analyzed the impact of the FSH level on the probability of ongoing pregnancy in a timedependent way, taking into account both age and whether or not treatment was used.

\section{MATERIALS AND METHODS}

\section{Patients}

All FSH values measured between July 1995 and July 1999 were obtained from the clinical chemistry laboratory database. A total of 5,040 results from 3,103 women were available. Since it is not a standard policy to repeat the measurement of basal FSH, the first FSH value was chosen if there was more than one result per patient. In most cases, FSH levels were reassessed because they were elevated. Although in many studies the highest FSH level is used because it is considered to be the most predictive $(15,16)$, we opted for the first one during the general infertility evaluation because management was based on that level.

The inclusion was stratified by three FSH classes, FSH $<10.0,10.0-15.0$, and $>15.0 \mathrm{IU} / \mathrm{L}$. The number of patients per FSH class in the total population is given in Table 1. As there were large numbers of patients in the first two FSH classes, a random sample was taken to keep the amount of work attainable. All patients with FSH above $15.0 \mathrm{IU} / \mathrm{L}$ were initially included, as is shown in Table 1.

The cutoff levels of FSH used in this study were adopted from the protocol used in our clinic, giving guidelines for management of patients with elevated FSH levels. Levels from 10.0 to $15.0 \mathrm{IU} / \mathrm{L}$ were considered as moderately elevated. The value of $10.0 \mathrm{IU} / \mathrm{L}$ using the World Health Organization Second International Reference Preparation 
(IRP) for human FSH (78/549) corresponds to $15 \mathrm{IU} / \mathrm{L}$ when measured with the former second IRP for hMG $(5,9)$. The cutoff value of $15.0 \mathrm{IU} / \mathrm{L}$ was chosen because in an earlier performed study IVF outcome in patients with a basal FSH above that value was poor (17). This cutoff value corresponds to $22.4 \mathrm{IU} / \mathrm{L}$ when measured with the IRP hMG. However, even when the same IRP is used, the application of different assays can lead to quite a lot of variation around the reference value of, for instance, $15.0 \mathrm{IU} / \mathrm{L}$ (18).

From these stratified groups, patients were selected who fulfilled the following inclusion criteria: $18-46$ years of age, two ovaries present, regular menstrual cycle between 21 and 35 days, and trying to become pregnant for at least 12 months. As the objective was to analyze spontaneous and treatment-related conceptions during the follow-up period, patients with occlusion of both tubes, with endometriosis stages III and IV according to the revised American Fertility Society classification (19), or with an azoospermic partner were excluded. Furthermore, the patients could not participate in an egg donation program.

Whether patients fulfilled the inclusion criteria was assessed on the basis of the computerized hospital information system and data in patient charts. The final numbers of patients satisfying these criteria are presented in Table 1. Far more patients had to be excluded in the group with FSH levels $>15.0 \mathrm{IU} / \mathrm{L}$ because the majority of these patients had an irregular cycle. Moreover, especially in the patients with the highest FSH levels, patients often were not referred for an infertility disorder.

\section{Infertility Evaluation}

The evaluation of new subfertile couples visiting our clinic has been described elsewhere (20). In addition, serum Chlamydia antibody testing and early follicular FSH measurements were routinely performed. If Chlamydia antibodies were present, a diagnostic laparoscopy was advised. In case of risk factors for tubal pathology, such as, for example, ectopic pregnancy or abdominal surgery in the past, but no Chlamydia antibodies present, hysterosalpingography (HSG) was performed. Patients with no risk factors for tubal pathology and absent Chlamydia antibodies were assumed to have a very low chance of tubal damage $(21,22)$, and further evaluation of tubal function was postponed or an HSG with oil-soluble contrast for therapeutic purposes was performed (23), as indicated by the guidelines of the Dutch Association of Obstetrics and Gynecology.

The management of subfertile couples was decided on the results of the diagnostic evaluation and the estimation of the probability of spontaneous conception (20). The medical protocol used in our clinic also gives guidelines for management of patients with elevated FSH levels. Patients with FSH levels of 10.0-15.0 IU/L received the usual management based on findings of the infertility workup and prognosis estimate, although they were sometimes advised to start treatment sooner. Patients with FSH levels above 15.0 IU/L were counseled as having poor chances to conceive. They were either excluded from infertility treatment or were advised to seek immediate IVF treatment. These decisions were based on clinical judgment after considering all data in the patient record.

\section{FSH Assay}

Serum concentrations of FSH were initially measured by the Ezymun-FSH test (Boehninger Mannheim, Mannheim, Germany) on the automated immunoanalyser ES-600. The observed interassay coefficients of variation were $3.7 \%$, $2.3 \%$, and $2.7 \%$, respectively, at $8.1,22$, and $75 \mathrm{IU} / \mathrm{L}$. After April 1996, FSH was measured in plasma by using the automated immunometric FSH assay by Chiron Diagnostics (Tarrytown, NY) on the automated ACS-180 immunoassay platform. For this assay, the coefficients of variation observed were $3.9 \%, 3.9 \%$, and $4.7 \%$ at $5.5,26$, and $64 \mathrm{IU} / \mathrm{L}$, respectively. Both assays were calibrated against the World Health Organization Second International Preparation for human FSH. The agreement between the two methods is given by the equation $\mathrm{FSH}_{\mathrm{ACS}}=.84 \times \mathrm{FSH}_{\mathrm{ES}-600}-0.31(r$ $=0.99, \mathrm{n}=100)$. Results obtained with the ACS were converted to the corresponding ES-600 values by this formula.

\section{Data Collection, Definitions, and Outcome Measures}

From the patients' charts, information on duration of infertility at the time of FSH measurement, type of infertility (primary/secondary), semen characteristics, tubal evaluation, type and start of treatment, occurrence of pregnancy, and pregnancy outcome was obtained. Patients were assigned to three diagnosis groups based on the main etiology of the fertility problem. Immunological factors and abnormal semen parameters were considered male factor. A small group had stage I or II endometriosis (19) and were considered as a separate group. If no obvious explanation was found, patients were considered as belonging to the group with unexplained infertility.

Patients who did not become pregnant and patients for whom the outcome of the pregnancy was not known were sent a questionnaire to obtain information on treatment, pregnancy, and outcome of pregnancy. A total of $82(67 \%)$, $70(56 \%)$, and $37(70 \%)$ patients were sent a questionnaire in the three respective FSH groups. From these patients, respectively, 45 (55\%), 34 (49\%), and 20 (54\%) returned it completed. Patients who were not sent a questionnaire were either pregnant or had moved to an unknown address. The patients not returning the questionnaire or with unknown addresses were considered not pregnant to avoid overestimation of pregnancy rates.

Patients included in the study were given a code to ensure anonymity. From patients willing to answer the questionnaire, written informed consent was obtained. The study was approved by the institutional review board. 
Patient characteristics and ongoing pregnancy rate of four FSH groups.

\begin{tabular}{|c|c|c|c|c|}
\hline Variable & $\begin{array}{c}\text { FSH }<10.0 \mathrm{IU} / \mathrm{L} \\
(\mathrm{n}=122)\end{array}$ & $\begin{array}{c}\text { FSH 10.0-15.0 IU/L } \\
(\mathrm{n}=126)\end{array}$ & $\begin{array}{c}\mathrm{FSH}>15.0 \mathrm{IU} / \mathrm{L} \\
(\mathrm{n}=53)\end{array}$ & $P$ \\
\hline Age (years) & $33.4(27.9-38.1)$ & $34.6(29.5-40.9)$ & $36.8(29.7-41.1)$ & $<.001^{\mathrm{a}}$ \\
\hline Duration of infertility (months) & $28(14-66)$ & $25(12-60)$ & $25(13-73)$ & $.45^{\mathrm{a}}$ \\
\hline Primary fertility disorder & $80(66)$ & $88(70)$ & $29(55)$ & $.15^{\mathrm{b}}$ \\
\hline \multicolumn{5}{|l|}{ Semen analysis } \\
\hline Morphology (\%) & $9(1-22)$ & $11(1-21)$ & $10(3-26)$ & $.15^{\mathrm{a}}$ \\
\hline \multicolumn{5}{|l|}{ Infertility diagnosis } \\
\hline Male factor & $85(70)$ & $74(59)$ & $28(53)$ & \\
\hline Unexplained & $34(28)$ & $45(36)$ & $23(43)$ & \\
\hline Endometriosis I/II & $3(3)$ & $7(6)$ & $2(4)$ & \\
\hline Follow-up period from $\mathrm{FSH}^{\mathrm{c}}$ & $15(3-38)$ & $12(1-43)$ & $10(1-51)$ & $.16^{\mathrm{b}}$ \\
\hline
\end{tabular}

Note: Values are median (10th-90th percentiles) or numbers (\%). NA = not applicable. Note that the sum of the median value of the duration of fertility and the median value of follow-up period from FSH measurement is not equivalent to the median value of total follow-up period from start of child wish; the sum of the mean values, however, is equivalent to the mean total follow-up.

${ }^{\mathrm{a}}$ Kruskal-Wallis test.

${ }^{\mathrm{b}} \chi^{2}$-test.

${ }^{\mathrm{c}}$ Follow-up from FSH measurement to ongoing pregnancy or last date of contact.

${ }^{\mathrm{d}}$ Follow-up period from start of child wish to ongoing pregnancy or last date of contact.

Van Rooij. Elevated FSH level and pregnancy. Fertil Steril 2004.

The outcome measure-ongoing pregnancy-was defined as a viable pregnancy of at least 11 weeks of gestational age. Besides looking at the overall ongoing pregnancy rate, a separate analysis of treatment-independent and treatment-dependent ongoing pregnancy was also performed as FSH levels influenced the start of treatment. Furthermore, the time to ongoing pregnancy was calculated from the date of FSH measurement to the date of conception leading to an ongoing pregnancy. Patients were censored at the last date of follow-up.

\section{Methods of Analysis}

Data were analyzed with SPSS 10.1 (SPSS Inc., Chicago, IL). Variables were tested for normality of distribution with the Kolgomorov-Smirnov test. As all variables, except age, were not normally distributed, the results are given in median and 10th to 90th percentiles. For comparisons of the three FSH groups, we made use of the Kruskal-Wallis test and the $\chi^{2}$ test whenever appropriate. Ongoing pregnancy rates, overall, treatment-independent, and treatment-dependent, per FSH group were calculated, and within these FSH groups for different arbitrary age categories. Treatmentdependent pregnancy rates were only calculated for the patients actually starting treatment $(n=188)$. We also looked separately at pregnancy rates of patients with extremely elevated FSH levels above 20.0 IU/L.

Pregnancy rates of the different age classes regardless of FSH were assessed separately, and these were corrected as the data sampling was stratified by FSH classes. Only random samples of respectively $6 \%$ and $44 \%$ were taken from the classes FSH $<10.0 \mathrm{IU} / \mathrm{L}$ and FSH in the range 10.0-15.0 IU/L, whereas all patients with FSH $>15.0$ IU/L were initially included. Therefore, there are relatively fewer patients with FSH $<15.0$ IU/L in the age classes than when a random sample out of the population had been chosen. For the three age classes, the number of pregnancies and the number of patients in each FSH subgroup were weighted by the inverse of the sample fraction of the subgroup to correct for the sampling design.

To correct for differences in the duration of follow-up, a Cox regression analysis was performed with time to overall ongoing pregnancy as the outcome. The contribution of the variables FSH (as a continuous variable), age, and the timedependent variable "being on treatment or not," which equals 0 when a patient is without treatment and 1 when the patient is being treated, were assessed in a univariate and multivariate analysis. We performed a bootstrap procedure to correct all survival analyses for the stratified design. $P<.05$ was considered statistically significant.

\section{RESULTS}

A total of 301 patients were included in the analysis. The patient characteristics per group are shown in Table 2. Patients with FSH levels below 10.0 IU/L were significantly younger than patients with higher FSH levels. No differences 
in duration of infertility or proportion of primary infertility were seen. Furthermore, no statistical differences could be demonstrated in the diagnosis of infertility and in semen parameters, but there was a trend to a higher proportion of male factor subfertility in the normal FSH group.

The outcome measure overall ongoing pregnancy declined significantly over the three respective FSH groups. When assessing separately the patients with more extreme FSH values above $20.0 \mathrm{IU} / \mathrm{L}$, a clear decline in the ongoing pregnancy rate $(4 / 25,16 \%)$ was observed, whereas 11 of 28 patients with levels between 15.0 and $20.0 \mathrm{IU} / \mathrm{L}$ (39\%) obtained an ongoing pregnancy.

As the FSH value could influence whether or not treatment was started (verification bias), an overview is given of the patient management in the different FSH groups to gain insight into the clinical decisions made (lower part of Table 1). The proportion of patients not pregnant before treatment increased somewhat in the respective FSH classes. Of these nonpregnant patients, a clearly lower proportion proceeded to treatment in the FSH $>15.0 \mathrm{IU} / \mathrm{L}$ class. If patients with highly elevated FSH levels started treatment, there was a tendency to start with assisted reproduction techniques (ART, including IVF and intracytoplasmic sperm injection), omitting the step of inseminations. ART, as a second-line treatment if inseminations were unsuccessful, was performed in all FSH classes.

A separate analysis of treatment-independent and treatment-dependent ongoing pregnancy was performed (Table 3 ). As age differed among the three FSH groups, pregnancy rates per age category are given. Treatment-independent pregnancies in this table include spontaneous ongoing pregnancies before start of treatment as well as spontaneous ongoing pregnancies after treatment had been initiated. In the group with normal FSH levels, five patients conceived spontaneously after treatment and this was the case for two patients with FSH levels between 10.0 and $15.0 \mathrm{IU} / \mathrm{L}$. No patients with an FSH level above 15.0 IU/L conceived spontaneously after treatment.

Treatment-independent ongoing pregnancy rates were about similar in the patient group with FSH between 10 and $15 \mathrm{IU} / \mathrm{L}$ compared with patients with normal FSH levels. In the patient groups with FSH levels above $15.0 \mathrm{IU} / \mathrm{L}$, a decline in pregnancy rates was present, although this trend was not significant $(P=.07)$. The pregnancy rate in the 25 patients with extreme FSH levels above $20.0 \mathrm{IU} / \mathrm{L}$ was again lower (4\%) than that of the 28 patients with FSH levels between 15.0 and $20.0 \mathrm{IU} / \mathrm{L}(14 \%)$.

Comparing the three FSH groups in the different age categories, the declining trend in pregnancy rates was not present or became inconsistent when age was below 31 or above 36 years, respectively. The observed number of ongoing pregnancies per age class regardless of FSH is also given in Table 3. The percentages were corrected for the
TABLE 3

Treatment-independent and treatment-dependent ongoing pregnancy rates in different age classes stratified by FSH classes.

\begin{tabular}{|c|c|c|c|c|}
\hline & $\begin{array}{c}\mathrm{FSH}<10.0 \\
\mathrm{IU} / \mathrm{L}\end{array}$ & $\begin{array}{c}\text { FSH } 10.0-15.0 \\
\text { IU/L }\end{array}$ & $\begin{array}{c}\mathrm{FSH}>15.0 \\
\mathrm{IU} / \mathrm{L}\end{array}$ & $\begin{array}{l}\text { All FSH } \\
\text { classes }^{\text {a }}\end{array}$ \\
\hline \multicolumn{5}{|c|}{ Treatment-independent ongoing pregnancy } \\
\hline Age $\leq 30$ & $29(12 / 41)$ & $26(7 / 27)$ & $25(2 / 8)$ & $29(21 / 76)$ \\
\hline Age $31-36$ & $28(16 / 57)$ & $22(13 / 59)$ & $5(1 / 22)$ & $27(30 / 138)$ \\
\hline Age $\geq 37$ & $8(2 / 24)$ & $15(6 / 40)$ & $9(2 / 23)$ & $10(10 / 87)^{\mathrm{b}}$ \\
\hline All ages & $25(30 / 122)$ & $21(26 / 126)$ & $9(5 / 53)^{\mathrm{c}}$ & \\
\hline \multicolumn{5}{|c|}{ Treatment-dependent ongoing pregnancy ${ }^{\mathrm{d}}$} \\
\hline Age $\leq 30$ & $62(16 / 26)$ & $56(9 / 16)$ & $75(3 / 4)$ & $61(28 / 46)$ \\
\hline Age $31-36$ & $56(20 / 36)$ & $45(17 / 38)$ & $33(5 / 15)$ & $54(42 / 89)$ \\
\hline Age $\geq 37$ & $65(13 / 20)$ & $28(7 / 25)$ & $25(2 / 8)$ & $59(22 / 53)^{\mathrm{e}}$ \\
\hline All ages & $60(49 / 82)$ & $42(33 / 79)$ & $37(10 / 27)^{\mathrm{f}}$ & \\
\hline
\end{tabular}

Note: Rates are in the form of percents.

${ }^{a}$ The pregnancy rates per age classes are corrected for the stratified sampling design.

${ }^{\mathrm{b}}$ All FSH classes, $\chi^{2}$ test for treatment-independent pregnancy rates differences in age classes, $P=.03$.

${ }^{c}$ All ages, $\chi^{2}$ test for treatment-independent pregnancy rates differences in FSH classes, $P=.07$.

${ }^{\mathrm{d}}$ Patient actually starting treatment, $\mathrm{n}=188$.

${ }^{\mathrm{e}}$ All FSH classes, $\chi^{2}$ test for treatment-dependent pregnancy rates differences in age classes, $P=.28$.

${ }^{\mathrm{f}}$ All ages, $\chi^{2}$ test for treatment-dependent pregnancy rates differences in FSH classes, $P=.03$.

Van Rooij. Elevated FSH level and pregnancy. Fertil Steril 2004.

stratified sampling during inclusion. The patients older than 36 years had a clearly lower pregnancy rate than the two younger groups $(P=.03)$.

Of the 188 patients followed after starting treatment, the treatment-dependent ongoing pregnancy rates became lower as FSH increased $(P=.03)$, but when comparing FSH classes per age group again this trend almost disappeared. Patients $>36$ years of age had clearly lower ongoing pregnancy rates during the treatment period when FSH was elevated, whereas in the younger age categories no clear trend of declining pregnancy rates with higher FSH levels was present. Three (27\%) of 11 patients with extremely elevated FSH levels ( $>20.0 \mathrm{IU} / \mathrm{L})$ even achieved an ongoing pregnancy during the treatment period. Patients with FSH levels between 15.0 and $20.0 \mathrm{IU} / \mathrm{L}$ had a similar pregnancy rate (7/16, 44\%) compared with patients with moderately elevated FSH levels (10.0-15.0 IU/L). The corrected pregnancy rates for the different age classes regardless of FSH were similar $(P=.28)$.

To correct for different times of follow-up, a Cox regression analysis was performed (Table 4). First, the influence of the variables FSH, age, and treatment on time to overall ongoing pregnancy was assessed in a univariate analysis. Both FSH and being on treatment or not were significantly associated with the time to pregnancy. Treatment improved 
Univariate and multivariate Cox regression analysis with time to overall ongoing pregnancy rate as outcome.

\begin{tabular}{|c|c|c|c|c|c|c|c|c|}
\hline \multicolumn{3}{|l|}{ Univariate analysis } & \multicolumn{6}{|c|}{ Multivariate analysis } \\
\hline Age (per year) & $0.97(0.91-1.01)$ & .17 & $\begin{array}{l}\text { FSH adjusted for } \\
\text { treatment (yes/no) }\end{array}$ & $0.96(0.90-1.01)$ & .08 & $\begin{array}{l}\text { Age adjusted for } \\
\text { treatment (yes/no) }\end{array}$ & $0.97(0.91-1.01)$ & .16 \\
\hline Treatment (yes/no) & $3.29(2.01-5.42)$ & $<.001$ & $\begin{array}{l}\text { FSH adjusted for age and } \\
\text { treatment (yes/no) }\end{array}$ & $0.97(0.91-1.01)$ & .22 & $\begin{array}{l}\text { Age adjusted for FSH } \\
\text { and treatment (yes/ } \\
\text { no) }\end{array}$ & $0.97(0.91-1.02)$ & .26 \\
\hline
\end{tabular}

Note: $\mathrm{CI}=$ confidence interval; $\mathrm{OR}=$ odds ratio.

Van Rooij. Elevated FSH level and pregnancy. Fertil Steril 2004.

the probability of ongoing pregnancy by a factor of 3 . Higher FSH levels were associated with lower pregnancy rates, confirming the results of Table 2. However, when the effect of FSH was adjusted for age or for being on treatment or not, the significance of FSH on the outcome variable (almost) disappeared. This phenomenon was even clearer when the effect of FSH was adjusted for both age and being on treatment.

\section{DISCUSSION}

On the basis of the results of the study, it can be concluded that subfertile patients with regular cycles and elevated FSH levels have lower pregnancy rates, especially when FSH levels exceed 20.0 IU/L. However, the overall ongoing pregnancy rate for patients with FSH levels above $15 \mathrm{IU} / \mathrm{L}$ is $\sim 28 \%$, which is quite acceptable.

Because of the retrospective character of this study one should be careful in interpreting the results. Patients with higher FSH levels were significantly older compared with normal FSH patients. It may be that more older patients and patients with higher FSH levels were refused treatment.

Moreover, the clinical decision to treat was also dependent on other patient characteristics, such as the semen parameters and duration of infertility. Therefore, the treatment-dependent pregnancy rates of patients with elevated FSH levels tend to be too optimistic: the couples with the poorest prospects may often have been counseled against treatment. Applying a more pessimistic scenario, assuming that all patients not becoming pregnant would have a zero chance of pregnancy if treated, the treatment-dependent pregnancy rates of patients with FSH level $>15.0-20.0 \mathrm{IU} / \mathrm{L}$ and above $20.0 \mathrm{IU} / \mathrm{L}$ would have been $29 \%$ and $13 \%$, respectively. It is plausible that the real percentages will be somewhere in between this pessimistic and the observed (too optimistic) pregnancy rate.

Another potential bias in this study is the low response rate to the survey. However, all patients not returning the questionnaire were considered as not achieving an ongoing pregnancy, which avoids overestimation of pregnancy rates. Furthermore, the response rate in the three FSH groups was similar. However, we cannot estimate whether the profiles of patients with normal and elevated FSH levels not returning the questionnaires were similar.

We chose to use the first FSH level in cases where more than one FSH measurement was carried out. Repeated assessment mainly took place in case of an elevated FSH level. The highest FSH level is regarded as more prognostic for the outcome of treatment $(15,16)$. For the reasons mentioned above and to make it possible to infer the results to any other subfertility population, we opted for the first measured FSH level. When the analysis was performed using the highest FSH level, the same results were obtained (data not shown).

Treatment had a positive effect on pregnancy rates, which was especially clear in women with FSH levels above 15.0 IU/L. This indicates that patients with elevated basal FSH and regular cycles should not be advised to refrain from ART. However, whether treatment with mild ovarian stimulation combined with IUI or IVF will indeed increase the prospects for patients with elevated FSH has not been confirmed in this or any other study.

As oocyte quality may not be severely influenced in elevated FSH cases $(18,24)$, the benefits of the treatment modalities mentioned may be valid also for these patients. On the other hand, since patients with high FSH levels more often have a poor response to ovarian hyperstimulation with gonadotropins $(8,18)$, treatment-dependent pregnancy rates may become so much decreased that continued treatment in IVF will be not justified in poor responders. Finally, patients with high FSH levels and otherwise good prognostic factors may have been more often treated, leading to an exaggeration of the difference in treatment-dependent and treatmentindependent pregnancy rates in these patients compared with normal FSH patients.

The rather high pregnancy rates of patients with $\mathrm{FSH}$ $>15.0 \mathrm{IU} / \mathrm{L}$ are in contrast with the results presented by Levi et al. (12). Their conception rate was only $5 \%$ in a general 
infertility population with FSH levels above 14.2 IU/L. As their population also included patients with irregular cycles, it may represent a group with more advanced stages of reproductive aging, leading to a much less favorable outcome. Indeed, in our study, a considerable part of the patient group with FSH $>15.0 \mathrm{IU} / \mathrm{L}$ had to be excluded because of irregular cycles. The combination of irregular cycles with high FSH levels is a reflection of premature ovarian failure, and those patients should only be offered oocyte donation. Furthermore, Levi et al. (12) provided no information on the way that high FSH levels influenced decisions on patient management and which patients were treated. In other words, it is very possible that high FSH levels automatically would lead to exclusion of patients from treatment thereby possibly reducing success rates.

In another population of subfertile patients with regular cycles, only minor differences in pregnancy rates could be detected between patients with normal $(\leq 10 \mathrm{IU} / \mathrm{L})$ and elevated $(>10)$ FSH levels (13). Even in the group with elevated FSH levels, no significant difference was found between the mildly and highly ( $>20.0 \mathrm{IU} / \mathrm{L})$ elevated FSH levels, although in the latter group the pregnancy rate was lower and assessed in only a small group.

Our results suggest lower overall pregnancy rates in patients with FSH levels $>15$ IU/L (Tables 2 and 4, univariate analysis), but this effect almost disappeared after correcting for age (Table 4, multivariate analysis). The combination of age and FSH seems to be important for the estimation of pregnancy chances.

This affirms the conclusions of some recent reports in the literature but is in contrast with some others. In one report, the pregnancy rate after six cycles of ovulation induction was four times higher in patients $<40$ years of age than in patients 40 years of age or older, while both had elevated FSH levels (14). In IVF populations, a moderately elevated FSH level had limited predictive value in young women (9) and age seemed a more important predictive factor for the occurrence of pregnancy than $\mathrm{FSH}(8,18,25)$. In contrast, it has recently been shown that younger IVF patients with either a poor ovarian response in IVF or elevated FSH levels performed as poorly as the older patients, although pregnancy rates in the older women with raised FSH levels may have been overestimated because of selection (10). Also, studies investigating general subfertility populations concluded that an abnormal FSH level was an unfavorable sign, regardless of age $(12,26)$.

Based on our study, FSH does not have an independent predictive effect on pregnancy rates in a general subfertility population but should be interpreted in the light of other clinical variables like age. The younger age group may contain patients with endogenously elevated FSH levels based on, for instance, genetic polymorphism of the FSH receptor or familial dizygotic twinning $(27,28)$. Younger patients with elevated FSH levels therefore should not be regarded as belonging to the group of patients with definitely diminished ovarian reserve $(18,24)$.

In contrast, patients of 40 years or older with elevated FSH levels are very much more likely to suffer from exhausted ovarian reserve but will already be considered as such on the basis of their age alone. How should the result of FSH assessment in a general subfertility population be used? In case of an elevated FSH level ( $>15.0 \mathrm{IU} / \mathrm{L})$, in the initial evaluation patients should be told that although the probability of conceiving is lower, it seems justified for first-try routine treatment instead of resorting to oocyte donation or adoption.

Patients with only mildly elevated FSH levels (10-15 IU/L) seem to have a good probability of achieving a pregnancy and should be counseled in the same way as patients with normal levels (9). If treatment is started, the response to maximal ovarian stimulation should be especially critically evaluated, as poor responders with elevated FSH levels seem to have lower chances of pregnancy (18). Furthermore, the FSH level should be regarded in view of other variables such as, for instance, age. For example, patients $>40$ years of age who also have FSH levels $>15.0$ IU/L have very low prospects for an ongoing pregnancy (29).

In summary, subfertile patients with regular cycles and elevated FSH levels have reasonable pregnancy rates, both from spontaneous conceptions as well as from ART. Therefore, they should not be automatically excluded from treatment based solely on elevated FSH levels.

\section{References}

1. Schwartz D, Mayaux MJ. Female fecundity as a function of age: results of artificial insemination in 2193 nulliparous women with azoospermic husbands. Federation CECOS. N Engl J Med 1982;306:404-6.

2. van Noord-Zaadstra BM, Looman CWN, Alsbach H, Habbema JDF, te Velde ER, Karbaat J. Delaying childbearing: effect of age on fecundity and outcome of pregnancy. BMJ 1991;302:1361-5.

3. te Velde ER, Pearson PL. The variability of female reproductive ageing. Hum Reprod Update 2002;8:141-54.

4. Muasher SJ, Oehninger S, Simonetti S, Matta J, Ellis LM, Liu HC, et al. The value of basal and/or stimulated serum gonadotropin levels in prediction of stimulation response and in vitro fertilization outcome. Fertil Steril 1988;50:298-307.

5. Scott RT, Toner JP, Muasher SJ, Oehninger SC, Robinson S, Rosenwaks Z. Follicle-stimulating hormone levels on cycle day 3 are predictive of in vitro fertilization outcome. Fertil Steril 1989;51:651-4.

6. Toner JP, Philput CB, Jones GS, Muasher SJ. Basal follicle-stimulating hormone level is a better predictor of in vitro fertilization performance than age. Fertil Steril 1991;55:784-91.

7. Bancsi LF, Broekmans FJM, Mol BW, Habbema JDF, te Velde ER Performance of basal follicle-stimulating hormone in the prediction of poor ovarian response and failure to become pregnant after in vitro fertilization: a meta-analysis. Fertil Steril 2003;79:1091-100.

8. Sharif K, Elgendy M, Lashen H, Afnan M. Age and basal follicle stimulating hormone as predictors of in vitro fertilisation outcome. Br J Obstet Gynaecol 1998;105:107-12.

9. Esposito MA, Coutifaris C, Barnhart KT. A moderately elevated day 3 FSH concentration has limited predictive value, especially in younger women. Hum Reprod 2002;17:118-23.

10. El Toukhy T, Khalaf Y, Hart R, Taylor A, Braude P. Young age does not protect against the adverse effects of reduced ovarian reserve-an eight year study. Hum Reprod 2002;17:1519-24

11. Barnhart $\mathrm{K}$, Osheroff $\mathrm{J}$. We are overinterpreting the predictive value of serum follicle-stimulating hormone levels. Fertil Steril 1999;72:8-9.

12. Levi AJ, Raynault MF, Bergh PA, Drews MR, Miller BT, Scott RT Reproductive outcome in patients with diminished ovarian reserve. Fertil Steril 2001;76:666-9. 
13. van Montfrans JM, Hoek A, van Hooff $\mathrm{MH}$, de Koning $\mathrm{CH}$, Tonch $\mathrm{N}$, Lambalk CB. Predictive value of basal follicle-stimulating hormone concentrations in a general subfertility population. Fertil Steril 2000; 74:97-103.

14. Check JH, Peymer M, Lurie D. Effect of age on pregnancy outcome without assisted reproductive technology in women with elevated early follicular phase serum follicle-stimulating hormone levels. Gynecol Obstet Invest 1998;45:217-20.

15. Martin JS, Nisker JA, Tummon IS, Daniel SA, Auckland JL, Feyles V. Future in vitro fertilization pregnancy potential of women with variably elevated day 3 follicle-stimulating hormone levels. Fertil Steril 1996; $65: 1238-40$

16. Scott RT, Hofmann GE, Oehninger SC, Muasher SJ. Intercycle variability of day 3 follicle-stimulating hormone levels and its effect on stimulation quality in in vitro fertilization. Fertil Steril 1990;54:297302.

17. Bancsi LF, Huijs AM, den Ouden CT, Broekmans FJ, Looman CW, Blankenstein MA, et al. Basal follicle-stimulating hormone levels are of limited value in predicting ongoing pregnancy rates after in vitro fertilization. Fertil Steril 2000;73:552-7.

18. Van Rooij IA, Bancsi LF, Broekmans FJ, Looman CW, Habbema JD, te Velde ER. Women older than 40 years of age and those with elevated follicle-stimulating hormone levels differ in poor response rate and embryo quality in in vitro fertilization. Fertil Steril 2003;79:482-8.

19. American Fertility Society. Revised American Fertility Society classification of endometriosis: 1985. Fertil Steril 1985;43:351-2.

20. Eimers JM, te Velde ER, Gerritse R, Vogelzang ET, Looman CW, Habbema JD. The prediction of the chance to conceive in subfertile couples. Fertil Steril 1994;61:44-52.
21. Land JA, Evers JL, Goossens VJ. How to use Chlamydia antibody testing in subfertility patients. Hum Reprod 1998;13:1094-8.

22. Mol BW, Dijkman B, Wertheim P, Lijmer J, van der Veen F, Bossuyt PM. The accuracy of serum chlamydial antibodies in the diagnosis of tubal pathology: a meta-analysis. Fertil Steril 1997;67:1031-7.

23. Johnson N, Vandekerckhove P, Watson A, Lilford R, Harada T, Hughes E. Tubal flushing for subfertility. Cochrane Database Sys Rev 2002; CD003718.

24. Lambalk CB. Value of elevated basal follicle-stimulating hormone levels and the differential diagnosis during the diagnostic subfertility work-up. Fertil Steril 2003;79:489-90.

25. Creus M, Penarrubia J, Fabregues F, Vidal E, Carmona F, Casamitjana $\mathrm{R}$, et al. Day 3 serum inhibin B and FSH and age as predictors of assisted reproduction treatment outcome. Hum Reprod 2000;15: 2341-6.

26. Scott RT, Opsahl MS, Leonardi MR, Neall GS, Illions EH, Navot D. Life table analysis of pregnancy rates in a general infertility population relative to ovarian reserve and patient age. Hum Reprod 1995;10:170610.

27. Lambalk CB, de Koning CH. Interpretation of elevated FSH in the regular menstrual cycle. Maturitas 1998;30:215-20.

28. Perez MM, Gromoll J, Behre HM, Gassner C, Nieschlag E, Simoni M. Ovarian response to follicle-stimulating hormone (FSH) stimulation depends on the FSH receptor genotype. J Clin Endocrinol Metab 2000;85:3365-9.

29. Watt AH, Legedza AT, Ginsburg ES, Barbieri RL, Clarke RN, Hornstein MD. The prognostic value of age and follicle-stimulating hormone levels in women over forty years of age undergoing in vitro fertilization. J Assist Reprod Genet 2000;17:264-8. 